\title{
The research and the development of the wide area relaying protection based on fault element identification
}

\author{
Xianggen Yin, Zhe Zhang, Zhenxing Li, Xuanwei Qi, Wenbin Cao* and Qian Guo
}

\begin{abstract}
The urgent problem of the relaying protection in the modern AC/DC hybrid connected grid and the development of the wide area communication, the information process and the intelligent technology powerfully promotes the development of the technology of the wide area relaying protection (WARP), which has become a research hotspot that attracts extensive attention. Originated from the basic concept of the wide area relaying protection, this paper analyses the advantages, the effects and the functions of the wide area relaying protection. The two main approaches to realize the wide area protection, which are on-line adaptive setting (OAS) principle and fault element identification (FEI), are introduced in this paper. Aimed at improving the performance of the backup protection, the research content and the technology demand of the wide area protection are proposed, meanwhile, the basic principle and the algorithm of the fault element identification are introduced. At last, the scheme of the limited wide area relaying protection based on the existing pilot channel of the main protection is discussed.
\end{abstract}

Keywords: Wide area protection, Wide area relaying protection, On-line adaptive setting, Fault element identification, The limited wide area protection

\section{Introduction}

Because of the extremely unbalance distribution of the electrical load and the energy in China, the Chinese power grids are required to transport the large-scale electrical power from the west to east over long distances, of which the scale is expanding rapidly, the structure is becoming more and more complicated, the technology continues to improve and the operation is flexible and changeable. Meanwhile, with the intensive construction of the large-scale ultra-high or extra-high voltage HVDC, the national grid is becoming an unprecedented large-scale AC/DC hybrid connected complex power system, of which the system protection is confronted with the severe challenges. If the fault cannot be cut-off in time, there will be a complex and evolutionary accident process in the AC/DC hybrid power system, and what's worse, the HDVC will be forced to quit operation, causing the huge power shortage and severely

\footnotetext{
* Correspondence: 1019097704@qq.com

State Key Laboratory of Advanced Electromagnetic Engineering and Technology, Huazhong University of Science and Technology, Wuhan 430074, China
}

threatening the safe and stable operation of the power grid. In order to cut-off the faults rapidly and guarantee the safe operation of the whole power grid, the protection of the $\mathrm{AC} / \mathrm{DC}$ hybrid system is required to operate faster, more reliably, more accurately and more sensitively, and especially, it is of great significance to increase the operation speed of the backup protection.

The operation of traditional relaying protection depends on the fixed value of off-line setting. However, in complex power system, especially when the operating mode of power system frequency changes, the cooperation and the coordination among the protections is hard to be achieved. For example, the selectivity, speediness and sensitivity of the protection cannot be satisfied at the same time. In the complex power system, because of the difficulty of the setting value calculation of the relaying protection, especially the backup protection, several backup protection configurations are trend to be simplified or abandoned. These conditions will increase the risk of the grid in urgent emergency. For instance, the clearing time of the fault will be prolonged and the isolation region of the fault will be extended, when the 
primary protection refuse to operate due to low sensitivity and the breaker fails to trip or loss of protection power, resulting in the local grid disaster. Another example is that, during the large-scale power flow transfers in the power system, the unexpected cascading tripping of the backup protection may occur, which is the main reason for the accident extension and even the large area blackout [1-3].

To improve the performance of traditional protection fundamentally and satisfy the safety protection requirement of the modern AC/DC hybrid complex grid, in recent years, with the development of wide-area information collection technology in power system, the researches on protection and controlling based on wide area measurement information (hereinafter referred to as wide area information) are becoming a focused research hotspot in the global power industry [4-6]. In China, many researchers have put their effort into the studies on the wide area protection. There is progress on the research of the basic principle and the implementation scheme for the wide area protection. Meanwhile, several local industrial wide area protection systems have been put into use in actual power grid.

This paper analyses the advantages, the effects and the functions of the WARP, from the basic concept of the WARP. The two main approaches to realize the wide area protection: the on-line adaptive setting (OAS) principle and fault element identification (FEI) are introduced in this paper. The research content and the technology demand of the wide area backup protection are proposed. The basic principle and the algorithm of the fault element identification are introduced. The scheme of the limited WARP technology with high reliability and speed based on the existing pilot channel of the main protection is discussed.

\section{The wide area relaying protection The challenge of traditional relaying protection in modern power system}

As the primary barrier of the power system, the relaying protection takes the responsibility of the fault clearing. However, with the development of the modern power system, the traditional relaying protection cannot satisfy the requirement for the safe and stable operation of the power system. The main problems are as follows:

\section{The difficulty in calculation and cooperation of the setting value}

The relaying protection system is mainly constituted by main protection and backup protection. The multi-stage backup protection mainly consists of the local backup protection and the remote backup protection of the power system, of which the typical mode is three stage protections. Because the grid structure and operation mode of the modern power system is becoming more and more complex and variable, the coordination among the related backup protection is very complex. Under such condition, the backup protection that only utilizes the local measurement and coordinate through the different time delay cannot satisfy the selectivity requirement of the power system protection. Because of the above problem, the backup protection trend to be simplified in the actual engineering application, and what's worse, the zone II backup protection is suggested to be cancelled and the zone III backup protection should be simplified in some situation. It is worth attention that when high resistance fault occurs in the large power system, the dual-configuration main protection still cannot detect the fault sensitively, which will prolong the fault clearing time and expand the scope of tripping. This will undoubtedly increase the risk of the local grid disaster under emergency state.

\section{Long time trip of remote backup protection}

The multi-stage cooperation mode will lead to a long delay time of the backup protection, easily resulting in commutation failure in power grid at receiving side, which is harmful to the security of AC/DC hybrid power grid.

\section{The lack of self-adaptive ability}

The setting coordination of traditional backup protection is based on the fixed operation mode of the power system. When the grid structure and operation mode of power grid changes, the backup protection may be hard to corporate and coordinate, which may cause the misoperation of the protection and expansion of the fault range.

\section{The potential risk of misoperation}

When the grid structure or running condition changes unexpectedly, the large load power flow transferring may occur in a large scale. The zone III distance protection may cascade trip unexpectedly, resulting in the system splitting or large scale blackout.

The major cause of these problems is that the relaying protection operates only based on the local information at the installation position. If more comprehensive information about the present power system can be acquired, for example, if the backup protection can get the information of the present system and the related information from the wider area, the performance of the protection will be greatly improved. For example, if the remote backup protection can get the relevant information from the remote protected device, the fault can be identified more accurately and cleared more quickly. The problems of traditional protection may be eliminated through the wide area information. 
Wide area protection and the wide area relaying protection Wide area protection (WAP) is the protective relay and the security and the stability control of the power system based on the wide area information, which consists of WARP and wide area security control (WASC) [7]. The wide area information is the information from the local and the remote power grid, rather than the information only from the protected device.

The narrow definition for the wide area protection is only related to the security and stability control, emphasizing the protection for the whole power system $[3,8$, 9]. There are also several literatures regarding the security control among $0.1 \sim 100 \mathrm{~s}$ as wide area protection $[10,11]$, in order to emphasize the protection for the wide area power system general safety. From the perspective of the history and applications, the stable control function is achieved by using the local information or wide area information. In recent years, the real-time wide area information are more and more used to improve the stable control function [10, 11], which has enriched the concept of wide area stable protection from the aspect of wide area information. In addition, several literatures, call the WARP as wide area back-up protection [3], which indicates that using the wide area information to improve the performance of backup protection is more concerned at present.

The relaying protection and security and stability control can achieve the security emergency control together for the power system. The relaying protection is called as equipment protection, and the security and stability control is often taken as system protection or SPS (Special Protection System or System Protection Scheme), RAS (Remedial Action Scheme) [3, 8, 9]. In the view of above, the relaying protection can be called as fault protection, and the security and stability control can be called as security and stability protection.

The essence of the security emergency control in Chinese power system is the three lines of defense [12, 13]. In the process of emergency control, relaying protection and security control operate independently, perform their own functions and their function cannot replace each other. It is worth attention that there are two basic principles in the security emergency control: the necessity for failure clearing and the priority of failure clearing. This is the duty and responsibilities of relaying protection and WARP. The fault clearing is the premise of effective security control. So the function of backup protection cannot be replaced by security control [3].

\section{The function and characteristics of wide area relaying protection}

The important difference between WARP and traditional relaying protection is using the wide area measured information. The traditional relaying protection mainly uses the local information, and at most uses the remote information which is extended from local protected equipment. Please note that the protected line of the remote backup protection is adjacent to the neighbor element and the state of this line, the previous operation result of the other protection (main protection) and the relevant remote backup protection are all related with the remote information. However, the tradition protection only can make judgments just according to the local measurement information. Hence, the information used for the traditional protection is not sufficient for the power system protection, which is the main reason for the existing deficiency of the protection. Therefore, the studies on WARP at present focus on the backup protection, especially the remote backup protection.

The wide area information can effectively improve the performance of the backup protection. The protective information in the faulty region can be acquired by the wide area communication systems. The fault element can be determined by the multi-information fusion. Then the backup protection can make the protection decision based on the fault element, and the coordination between the protections can be simplified. Meanwhile, the real time system structure and the operation mode can be acquired through the wide area measurement and the communication technology. The setting value of the protection can be up-dated based on the current status of the power system. Hence, the sensitivity and the selectivity can be improved.

The farthest protection scope of relaying protection is the next adjacent equipment, which is the region of backup protection. Therefore, the protection scope of the relaying protection is limited. Hence the information of the WARP is limited, which introduces the concept of limited WARP. The most important characteristic of limited WARP is that it only needs the measurement information from the adjacent limited area, which can lighten the burden of wide area communication or information processing, which is beneficial to the realization of WARP. In practical engineering, it is possible to realize the limited-wide-area relaying protection by using the existing optical fiber communication channel of the pilot protection. On the other hand, the system protection is oriented for the safe and stable operation of the entire power system, which needs the information from the wide-range of the whole system. This is another important difference between the protective relay and the system protection.

Besides, in a substation, if not only the information of local protected equipment can be used, but also the information of relevant equipment in the station can be used, the performance of relaying protection can be improved effectively. This is called substation area relaying 
protection, which uses the substation area measurement information. The substation area protection does not need the remote communication, which is feasible for the industrial application, especially for the digital substation. The sharing and the utilization of the substation area information can improve the performance of the relaying protection and control functions in the substation, integrate the substation relaying protection and simplify the relaying protection configuration, extent the new relaying protection function flexibly, such as the bus-bar protection and the breaker failure protection in the medium or low voltage substation, implement the coordination between the relaying protection and the control and the unified substation protection system. Meanwhile, the substation protection system can access to the wide area protection system as the child-station, and corporate with the wide area protection to achieve the protection and control for the regional power grid. Therefore, some scholars have proposed the concept of the hierarchical protection, which is constructed by the bay layer relaying protection (traditional primary relaying protection), substation area layer relaying protection (substation area protection) and wide area layer relaying protection (WARP), of which the main design is shown in Fig. 1.
In Fig. 1, the bay layer relaying protection is only aimed at single equipment bay. With the information in bay unit, it achieves the protection function of devices in bay unit. It also collects information and executing orders for WARP. Substation area layer relaying protection is aimed at substation. With synthesized information from multiple equipments in the substation, it judges, decides uniformly and achieves protection and controlling function in substation area. It also preprocesses information and provides communication service for WARP. Wide area layer relaying protection is aimed at limited area grid. With synthesized information from multiple substations, it judges, decides uniformly and achieves wide area protection function in wide area.

\section{The basic approach to achieve the wide area relaying protection}

At present, there are mainly two different approaches to realize the basic function of WARP. One approach is based on the principle of online adaptive setting (OAS); another is based on fault element identification (FEI) to achieve WARP [7].

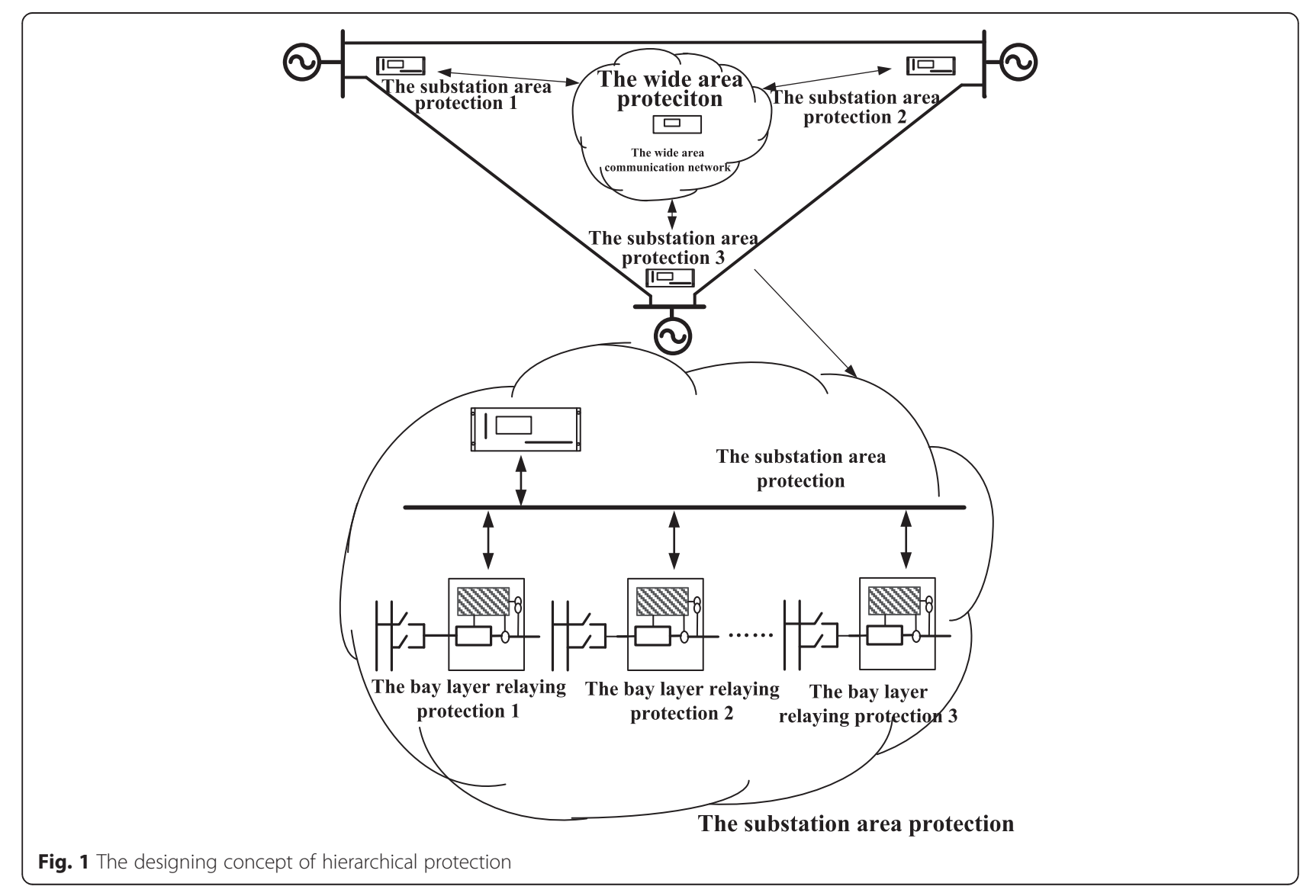




\section{The wide area relaying protection based on on-line adaptive settings}

The study on on-line adaptive setting (OAS) started at 1980s and the foreign scholars have defined it as following [14]: An on-line activity that modifies the preferred protective response to a change in system conditions or requirements. It is usually automatic, but can include timely human intervention. And several Chinese scholars express it as following [15]: Taking the eventtriggered mode, tracking the change of power system operation mode in time, calculating and on-line regulating the setting value of protection to prevent the protection from losing coordination and improve its sensitivity.

In recent 20 years, the researches on OAS setting approach are mainly around the fault disturbance domain identification, the search of minimum cut point and quick short circuit calculation and so on [15-18]. In China, the protection information system for the setting values online check has been constructed in the power system. Based on the wide area communication system, the protection information system can acquire the intime operation condition of the power grid, check and reset the setting value, and transfer the updated setting value to each protection device [19-22].

WARP based on OAS has been studied for a long time. There are several progresses achieved. But its application is limited. This is because, although this approach improves the sensitivity and selectivity by adjusting the fixed value online, it still cannot overcome the shortcomings of the traditional backup protection, including the difficulty of the setting value coordination, the long operation time delay and so on. This shortcoming is also the important reason for hidden failure of the backup protection, which leads to the cascading trip and threats the system safety. In addition, how to ensure the timeliness and reliability of the wide area information communication, and how to calculate the setting value quickly after the change of the operation mode are still the questions to be solved.

\section{The wide area relaying protection based on fault element identification}

The study on WAPR based on FEI begins at the end of 1990s. For example, Yoshizumi Serizawa came up with the wide area current differential backup protection [23] at 1998, which form the scope of differential protection according to the area of backup protection and it can identify the fault element accurately and determine the operation scope of backup protection.

The WARP based on FEI can be described as following: By using the wide area multi-point measurement information in the power system and taking several fault discriminate mechanism, the position of fault element and the state of fault clearing can be determined. And then, the cutting point of WARP can be determined. The breakers at the cutting point operate to isolate the fault through bay layer's equipment. Its superiority is that there is no need for setting calculation, the selectivity of backup protection can be guaranteed only by simple coordination of time and logic and the operation time of back up protection can be shorted effectively. For example, no matter where the remote backup protection locates, because it only coordinates with near backup protection (or main protection), the coordination between protection is simplified. Meanwhile, the backup protection can be prevented from cascading tripping during large scale load transferring.

The WARP based on FEI doesn't require the information changes in time of entire power system. Even for the remote back up protection, it only needs the relevant fault information of the nearby equipment which is in the rim of adjacent substation groups (the scope may be expanded appropriately from the perspective of information fault tolerance). Therefore, this is a limited wide area protection and it is beneficial to the achievement of its engineering.

Recently, the WARP based on FEI has drawn the research concern. There are many achievements in the aspect of identification of fault elements, system constitution and the industrial test. However, according to the requirement of engineering application, there are many technical problems remain to be solved. The following will discuss the topic.

Aiming at the potential risk problems that the unexpected cascading operation of backup protection which is probably caused by large scale load transfer, it can use the wide area information to analyze and distinguish the state of the load transferring in power system and take the measures [24, 25] such as, blocking or changing action characteristics for the relevant backup protection and so on, which can avoid the cascading trip of backup protection and guarantee the system security.

\section{The research contents of wide area relaying protection based on fault element identification} System constitution and zone-division technology WARP system has three basic links: 1) information acquisition and operation; 2) communication networks and information transferring; 3) fault element identification and action decision-making. In the whole power grid, based on the substation distribution situation, different system structures can be formed by reasonably distributing and organizing these three links, which are also accordant with the hierarchical protection structure. The first link is located at the bottom of the protection system which can be implemented by the intensively configured intelligent electric device (IED). The second link is the wide area communication network, which 
transfers the information between the first and third level. The third link makes the protection decision, of which the configuration form determines the wide-area protection system structure.

The system structure type of FEI-based WARP includes three main types: the centralized structure, the distributed structure and the distributed-centralized hybrid structure [26].

The distribution-centralized hybrid structure divides the protected zone of the power grid, every divided protected zone has a decision-making center which is responsible for the wide-area relaying protection and the information exchanges with the adjacent protected zone. This structure combines the advantages of the former two structures, and has a better research prospect. The zone-division distribution-centralized structure proposed in the article [27] is a typical hybrid structure. Hybrid structure needs to solve the issue of dividing the protected zone of the power grid, including the principle and method of automatic zone-division, the selection of the main substation, the boundary processing technology of the adjacent protected zone and so on $[27,28]$.

\section{The principle of fault element identification (FEI)}

The mechanism of fault element identification is the critical technologies for the FEI-based WARP. Consequently, the principle and the algorithm of fault element identification based on wide area information are the research hotspot in recent years, which mainly includes two aspects.

The first aspect is the research of the basic principle of fault element identification. In early time, several backup protection principles are proposed, such as the wide area current differential protection, wide area direction directional pilot protection [23, 29-32]. The wide area current differential backup protection is a kind of current differential protection utilizing the multi-side current in the protected region, of which the protected zone can cover the region of the local and the remote backup protection. This protection only needs a simple time delay cooperation between each protection, and can effectively guarantee the selectivity and the action speed of the backup protection. The wide area current differential protection has a simple and clear principle, and can detect various types of internal fault. But it relies on high precision synchronous sampling and still has some problems concerning with the threshold value setting method and the sensitivity of the protection, such as the wide area measurement error, the capacitance current compensation, and the high resistance grounding fault. The wide area directional pilot protection needs small communication traffic, and has low requirement for the synchronization sampling. However, the directional element is highly affected by high resistance grounding, open-phase operation and fault transition and so on. To overcome these problems, several principles of FEI based on fault voltage distribution, wide area integrated impedance or integrating variety of information are presented in recent years.

The second aspect is to improve the reliability of FEI by using multi-source information fusion. The process of distributed collection, decentralized processing and remote communication of the wide area measurement information may cause the information error. On the other hand, the WARP can acquire multi-source information from the protected zone, such as the electric quantity, the state quantity and even the operation results of the protection criterion. This multi-source information will be processed by using the method of artificial intelligence, such as the genetic [33] and the probability identification [34]. The adverse influence of bad data can be avoided and the error-tolerance of decision-making can be realized.

\section{Other technology issues}

A brief description of some important research issues are briefly introduced in the following:

\section{Tripping strategy}

After the fault elements are determined, the action status of breaker connected to fault element also need to be monitored. Only when a breaker refuse to work, can the wide area backup protection (far backup) trip the remote breakers that is associated with the faulty breaker., The wide area backup protection should cooperate with the other protection and the breakers, and trip the minimum breakers to isolate the fault.

\section{Communication technology}

Communication technology of the wide area protection involves two aspects: the communication in the substation area and the wide area communication. With the development of the digital substation technology based on IEC61850, the substation-area communication already has a perfect scheme and will be continuously improved.

As for the wide area protection communication, the region is wider, the distance is longer and the data flow is larger. The communication notes and links are numerous. The wide area protection communication has to bear the different kinds of the information exchange, such as the periodicity, the stochastic and the burst information, and the high-precision clock synchronization is of great importance. Recently, the PTN (Packet Transport Network, PTN) communication technology has become a widely-used communication technology in the telecom and industry, and has been applied in the power 
system. PTN can achieve the flexible transmission for the non-fixed granular business, overcoming the shortcomings, such as the low frame utilization of the SDH network, the poor safety of the GPS synchronization and the in-adaptation for the burst communications of the protection. PTN can get the target of the high real-time performance, reliability and security for the wide area protection. In addition, some scholars also study on the application of the WAMS (Wide Area Measurement System, WAMS) for the wide area protection [35-37].

Except the above content, the research subjects on the communications for WARP also include the structure model of wide area communication system, the analysis and control of communication traffic, the error information analysis, and the intelligent error-informationtolerant method.

\section{The problem of wide area information synchronization}

At present, this problem is solved by global satellite synchronous clock like GPS and Bei Dou. However, due to the natural and technological factors, the performance of this approach still needs to improve. Therefore, the principle and algorithm of wide area protection need to be concerned besides wide-area information synchronization performance.

\section{The interoperability of wide area protection device}

In order to implement the interoperability of wide area protection device, the studies and the practice on the IEC61850 standard for wide area communications are necessary in order to realize the plug and play of all wide area protection.

\section{Better configuration of backup protection system}

The wide area communication failures and the sensitivity of the wide area backup protection should be taken into account. To achieve a better configuration of backup protection system, the misoperation of the wide area backup protection should be prevented. The remote back-up protection based on local information with the fixed setting value, the high sensitivity and the long time delay, still needs to be reserved for the last level of the backup protection.

\section{Reliability and risk assessment of wide area relaying protection}

Since the wide area backup protection using multisource information from the wide area communication system, its reliability is an important issue. The multisource information is benefit to improve the reliability of WARP. The Reliability and risk assessment of WARP can provide an effective guide for the actual application of WARP [36].

\section{New principles of fault element identification}

The following briefly describes several new principles of FEI. They involve the basic principle and the multisource information fusion. Several aforementioned problems are considered when designing the protection algorithm.

\section{The principle of fault element identification based on fault voltage distribution}

There are several principles of FEI for a single element (for example a transmission line), such as the current differential, the pilot direction and the pilot distance, etc. Obviously, the current differential protection is strict with the synchronous sampling and difficult for the wide area protection. Meanwhile the performance of the pilot direction protection and the pilot distance protection is still non-perfect under the condition of complex faults. The principle of FEI based on the fault voltage distribution can both solve the above-mentioned two problems [35, 37].

This principle uses the measured fault component voltage and current on one side of the transmission line to estimate the fault component voltage on the other side. Hence, both of the measured and the estimated fault component voltages on two sides of the transmission line can be acquired. The measured value and estimated value of fault component voltages on both sides of the line are consistent, when external faults occur. As for internal faults, there exists some difference between the measured value and the estimated value of fault component voltage. So the faulty element can be identified based on the ratio between the measured values and the estimated values. Figure 2 is the distribution diagram of the measured value and estimated value of fault component voltage.

The principle of FEI is applicable to all types of fault components, including positive-, negative-, and zerosequence fault components. By the comprehensive utilization of six components combination criterion, this FEI method can effectively deal with the single-phase-toground fault, the asymmetric interphase fault and threephase-short-circuit fault.

Because only the amplitude of remote voltage is acquired for this wide area protection, this FEI method has low requirement for the wide area data synchronism, and only needs the synchronization correction based on the fault characteristics on the both sides of the line at the moment that the fault occurs in the line. The simulation calculation shows that this principle can correctly identify the fault line in the conditions of the high resistance grounding, weak feedback, faults on twophase operation of the line, evolved faults or fault during oscillation, and is not affected by power flow transferring. 


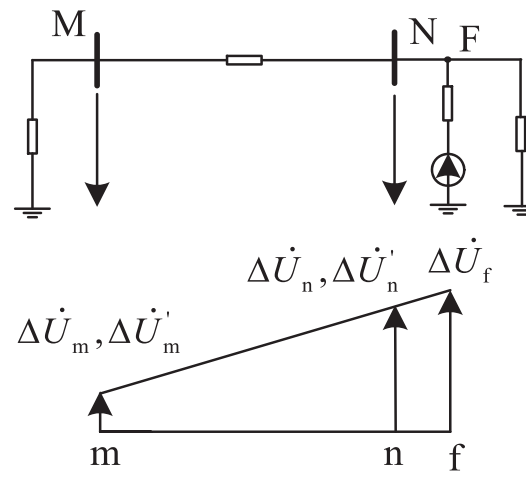

(a) The external fault
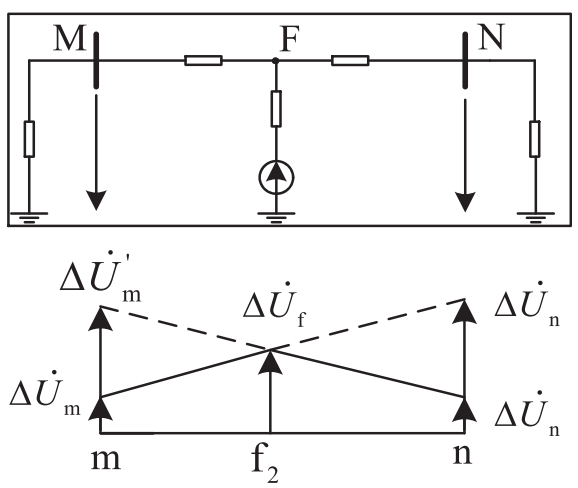

(b) The internal fault

Fig. 2 Distribution of fault components of voltages

The principle of fault element identification based on wide area integrated impedance

The wide area current differential protection is more susceptible to the line distributed capacitor than the common current differential protection, resulting in the lower sensitivity. This is because the number of lines under different operation condition is variable in the region of the wide area current differential protection. The distributed capacitance and the capacitance current may change in a wide range. In addition, it is more difficult to estimate and compensate the capacitance current in the wide area region of the differential protection. The pilot protection based on integrated impedance can overcome the influence of distributed capacitance and increase the sensitivity [38]. Introducing the concept of integrated impedance to wide area protection, the principle of FEI based on wide area integrated impedance can be established [39], which can overcome the defects of the wide area current differential protection.

The principle uses the multiport voltage and current in a region to form the integrated impedance, as shown in Fig. 3 . The identification of wide area integrated impedance is given by

$$
Z_{c d}=\frac{\dot{U}_{c d}}{\dot{I}_{c d}}=\frac{\sum_{i}^{N} \dot{U}_{i}}{\sum_{i}^{M} \dot{I}_{i}}
$$

Where, $M$ and $N$ are respectively the number of current that flow into the wide area relay protection area and the bus number on the region of the wide area relay protection.

When external fault occurs, the wide area integrated impedance reflects the equivalent line capacitive reactance of protection zone, of which the imaginary part value is thousands of ohms and the impedance angle is about $-90^{\circ}$. As for the internal fault, the wide area integrated impedance is related to factors, such as the system impedance, the line impedance and the fault resistance, which appears to be inductive reactance and has small imaginary part. Based on the difference of the integrated impedance when the internal fault and the external fault occur, the characteristic of the proposed protection is shown in Fig. 4. Simulation verifies that the algorithm has high sensitivity and is capable for the earth fault with high fault resistance. This algorithm is not affected by the capacitive current, two-phase operation of the line, evolved faults and system oscillation, meanwhile, has the advantages of phase selection.

\section{The limited wide area protection}

As mentioned before, the limited wider area protection that is based on the limited information can identify the fault element and improve the performance of the backup protection, meanwhile, can also speed up the remote backup protection under the condition of the substation DC power supply failure. The advantage of the limited wide area protection lies in that only the limited information in the adjacent region is needed, reducing the information communication and processing burden. Under the current technology condition, the existing

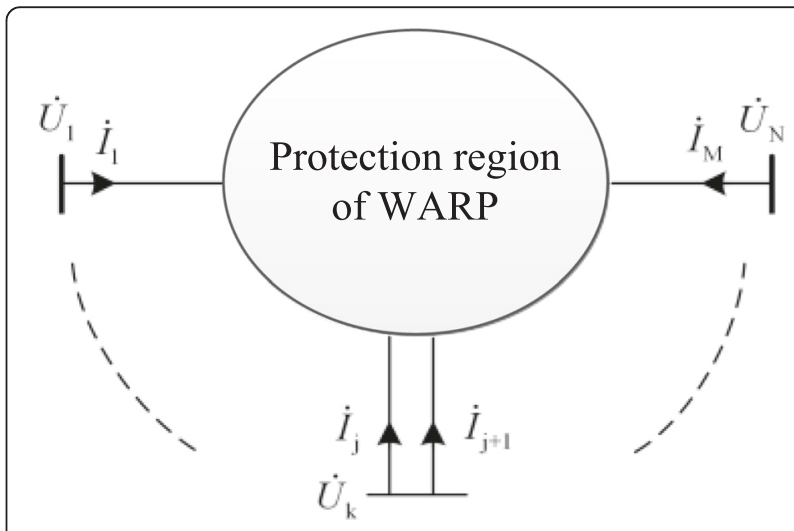

Fig. 3 Protection region of WARP 


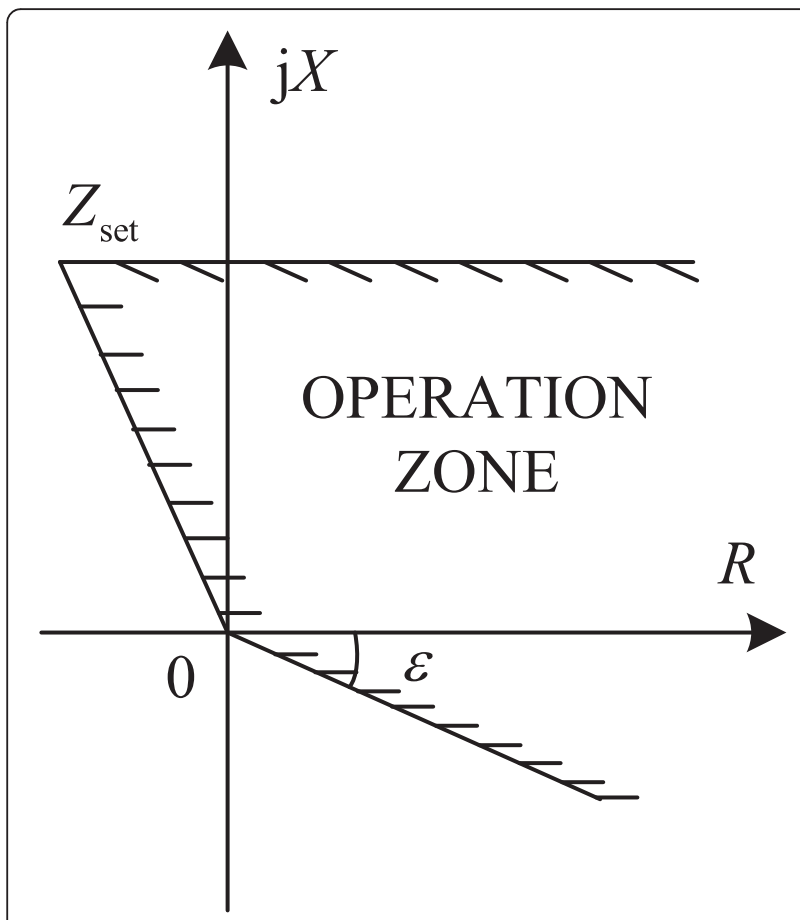

Fig. 4 Operation characteristics of WARP based on wide area integrated impedance

pilot channel of the current differential protection can be applied to transfer the protection information among the neighbor substations. Based on this, the limited wide area protection could be implemented.

Recently, the limited wide area has become a research hotspot due to its extensive application prospects. Some prototyping system has been put into operation in Chinese grid. The following introduces the principle and the application of some typical limited wide area protection, including the backup protection based on the pilot channel and the substation dc power supply failure protection.

\section{The limited wide area backup protection based on pilot protection channel}

According to the construction model of mentioned hierarchical protection, the bay layer protection is main protection. In addition, the substation area protection system is constructed inner the substation to measure and collect the internal substation electric information independently, which can achieve the backup protection function of connected lines and components. Combing the station area protection system and inter-substation communication channel, the simplified limited wide area protection can realize the protection information communication of each adjacent substation, which can identify the fault component and improve the action performance of backup protection. In this scheme, the backup protection (Including the zone-II and zone-III) of each line all takes the setting method that ensure the sensitivity of terminal protection scope, and the selectivity of the protection can be ensured by the inter substation communication. This method can simplify the setting and coordination of the backup protection, which can also accelerate the backup protection operation.

The following takes zone-II distance protection and zone-III distance protection for example which is shown in Fig. 5 to introduce the limited WARP based on intersubstation communication. This protection scheme needs two signal transmission, which respectively achieve the near backup protection and remote backup protection.

The first signal transmission: the zone II distance protection of the fault line immediately startup, and the adjacent normal line zone II distance protection is blocked. When the fault occurs in the line, the substation zone II distance protection detects the fault line and sends the judgement conclusion to the adjacent substation by the inner-substation protection channel: Send the information 'the fault occurs in this line' to the opposite station of the fault line and sent the information 'the fault occurs in the other line' to the other adjacent substations. Only when the substation area protection judges the position of fault in the protection region of the zone-II distance protection and the received the the fault occurs in this line' information from adjacent substation, the line can be determined as the fault line which can be cutoff by the zone II distance protection quickly. For example, when the fault occurs in the line L3 in Fig. 5, the distance zone-II of the protection 5 and protection 6 on the both sides of L3 will operate and receive the 'fault occurs in this line' information from the neighbor substation. Hence line L3 is taken for the fault line and can be cutoff fast. The distance protection 3 and 8 may also operate during the fault of line L3 because of the setting method that ensures the sensitivity of terminal protection scope. However, the protection 3 and 8 will receive the information 'the fault occurs in the other line' from the opposite substation, the line L2 and L4 cannot be judged as fault line. Hence, the fault line can be effectively judged based on the information between the adjacent stations, which will ensure the fast operation speed and the selectivity of the zone II distance protection.

The second signal transmission: When the zone-II distance protection operates, the relevant remote backup protection that is adjacent to the fault line is started-up. The signal for the first time identifies the fault line and determines all the relevant remote backup protection of the fault components. When the zone-III distance protection judges the fault occurs in the protection region and receive the startup signal from the neighbor substation, the zone-III distance protection will operate and 


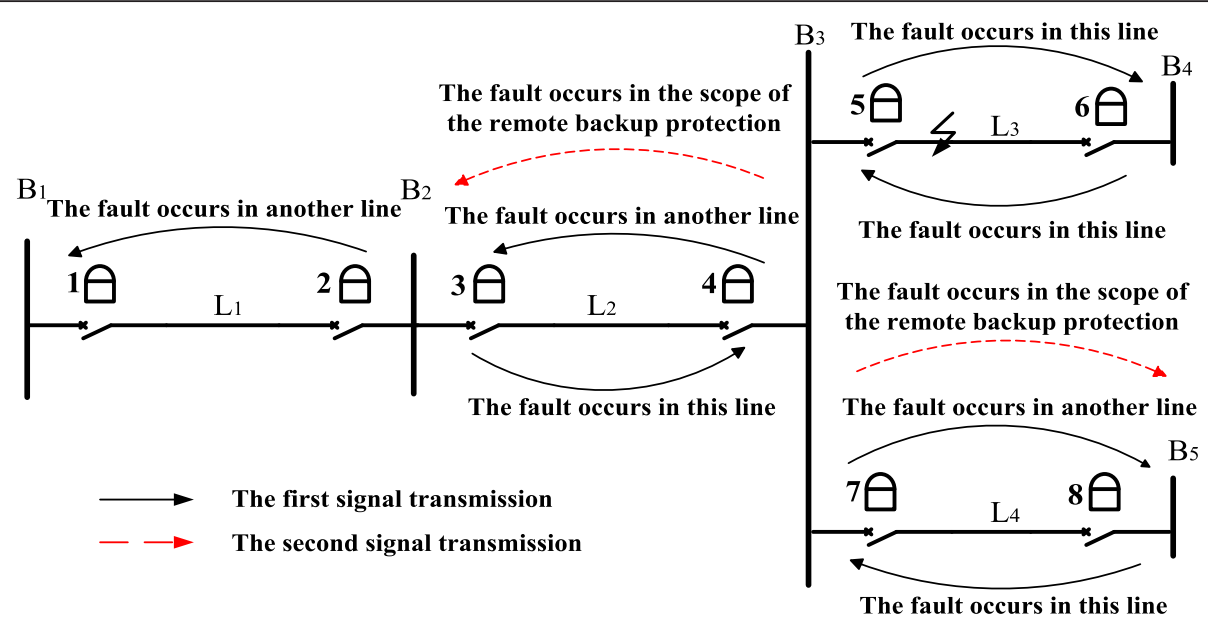

Fig. 5 Collaboration diagram of substation area backup protection information

cutoff the fault fast with short fix time delay. Taking the fault occurs in the line L3 in Fig. 5 as example, the protection 1,3 , and 8 will operate because of the setting mode that ensures the sensitivity of terminal protection scope. But, only the protection 3 and 8 will receive the startup signal and operate and the protection 1 will not misoperate.

Based on the above backup distance protection of inter-substation communication, the backup distance protection can take the invert setting mode to ensure the sensitivity and there is no need to coordinate. The operation time of the zone III distance protection can be set by the fixed time (such as $1 \mathrm{~s}$ ) delay, without the multi-step time delay, which can speed up the action speed. In addition, when the large scale power flow transfer occurs, because of the lack of the startup signal, the remote backup protection will not misoperate.

In the same way, utilizing the zero-sequence direction element, the limited wide area backup protection can be established based on the pilot channel. The high sensitivity and fast protection for the high resistance grounding fault can be achieved.

This protection scheme takes independent substation protection system to realize the protection function of backup protection. When the bay layer main protection fail or refuse to operate, the scheme can achieve the selective and fast protection of backup protection. For the $110 \mathrm{kV}$ or below $110 \mathrm{kV}$ system which doesn't configure the breaker failure protection, this scheme can achieve the function of fast remote backup protection when the breaker is fail.

\section{The limited wide area protection based on pilot} protection channel and the bay layer protection In the high- or ultrahigh- voltage system, the bay layer protection is configured with the current differential protection and backup protection based on the fiber pilot channel. By using the existing the bay layer protection as well as pilot channel between substations, the function of the limited wide area backup protection shown in Section V. A can also be realized.

The limited wide area backup protection based on the pilot channel and the bay layer protection is made up of the bay layer protection and decision unit of substation area protection. The backup distance protection of the bay layer protection for each line can be directly set based on the sensitivity and operation with the fix short time delay. It is not necessary for each protection to coordinate. The substation layer protection decision unit can acquire the protective results of the layer protection in the substation through the GOOSE network and that in the neighbor substation by the pilot channel. The protection scheme needs two signal transmission to realize the identification of fault element, which can respectively achieve the near backup protection and remote backup protection. Then startup the bay layer backup protection related to the fault element to isolate the fault.

In this scheme, the operation results of the bay layer protection in this substation and neighbor substation are acquired through the existing bay layer protection and pilot channel. The identification of fault element can be realized by the logic information of operation results. So this scheme is feasible for the implementation.

In China, the configuration of the protection in the ultra-high voltage system with the voltage grade of $220 \mathrm{kV}$ and above is completely dual. The signal acquirement system, the power supply of the protection device, the pilot channel and the trip-ping loop of each protection are all dual-configured. Hence, the limited wide area backup protection based on pilot channel has high reliability. 
The countermeasure for the failure of substation main power based on the limited wide area protection technology

The substation main power supplies the power for the control and protection devices and the operation loops. The main power failure in a substation occurs, the protection device and operating circuit cannot operate, which is the most severe accident in the substation. Constrained by the construction and operating principle (include the battery system), the operation statistics indicates that the failure of substation main power frequently occurred. This fault is especially severe in the substation supplied by single main power. The fault which occurs in substation and its line can be cleared only by the remote backup protection in neighbor substation. Therefore, it is of great importance for avoiding the expanding of accident to effectively determine whether the main power of substation is failing and to accelerate the remote backup protection when faults occur in the no-power substation.

\section{The basic principle of substation dc power supply failure protection with the help of communication}

In the substation, there is usually another communication power used for the remote communication except main power. These two kinds power is independent and backup of each other. Hence the substation area protection system can be supplied by both the main power and communication power. The system can monitor the running state. If the main power is lost, substation area protection system can be supplied by communication power and send the signal of power failure. When faults occur in the no-power substation, substation area protection will send tripping signal to adjacent substation, accelerating the speed of remote backup protection in adjacent substation. So the fault can be quickly isolated.

\section{Example of substation dc power supply failure protection} with the inner substation communication

Based on the above principle, a power supply wiring of substation area protection is shown in Fig. 6. This protection device is supplied by main power and communication power. A low-voltage relay is installed in the main power source to monitor the voltage. Once the main power failure occurs, the low-voltage relay operates and the signal will be sent to the substation area protection for the DC power failure judgement. In addition, because of the failure of the main power, the other protection device in the substation will not work. Protection device in the adjacent substation will send channel abnormity signal to the no-power substation because of the communication interrupt. Therefore, substation area protection in the substation without main power can comprehensively judge the loss of main power by collecting channel abnormity signal in adjacent substation and the operation signal of DC power lowvoltage relay.

When the main power fails, the substation area protection device supplied by communication power can still work normally. The differential current protection of transforms and bus in the no-power substation still can work and identify the fault. But the breaks in no-power cannot operate to cut-off the fault. In this case, the substation area protection will send tripping signal to adjacent substation to accelerate the isolation of the faults.

Because of some special reasons, such as the voltage measurement is lost, the substation area protection in the no-power substation cannot identify the line fault. The line fault can be identified by collecting the operation performance of pilot impedance component at another side of the line. If the pilot impedance component of the adjacent substation operates meanwhile the breaker is in off-state, the no-power substation will determine that fault occurs in this line, and send tripping signal to adjacent substation to remove fault promptly.

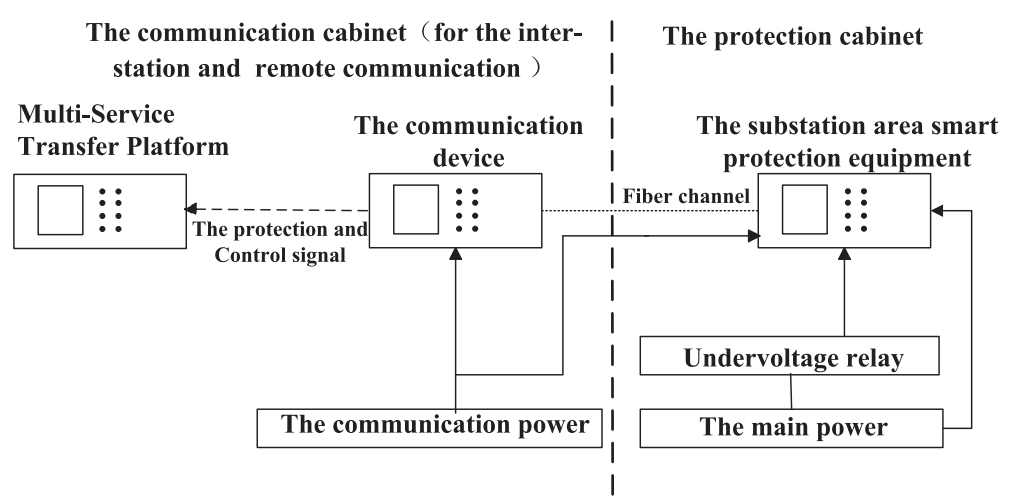

Fig. 6 Connection diagram of power for wide area protection equipment 


\section{Other special issues}

In the digital substation, the power of the SV and GOOSE network is supplied by the main power of the substation. Under the condition of the main power failure, the substation protection cannot acquire the protection information, and the fault in the no-power substation cannot be detected. Under such condition, the communication between the substations around the no-power substation can achieve the fault element identification, enhancing the performance the remote backup protection. This problem will be discussed in other article.

\section{Conclusion}

The urgent problem of the relaying protection in the modern AC/DC hybrid grid and the development of the wide area communication, the information process and the intelligent technology powerfully promotes the development of the technology of the wide area protection. Wide area protection includes wide area relaying protection (WAPR) and wide area security protection (WASP). At present, the research of WARP is focus mainly on wide area backup protection. WARP, used to clean fault, belongs to the first defense of three defense of security emergency control, which cannot be replaced by security emergency control. The farthest region of the wide area backup protection is the range of remote backup protection. Hence wide area backup protection has limited zone in respects of the information and action.

There are two main approaches to realize WARP, which are based on on-line adaptive setting (OAS) principle and fault element identification (FEI) principle. Some achievements have been obtained in these aspects currently, and several test systems have been put into use in the actual grid.

The paper discusses the research of wide area backup relaying protection, including the system constitution and zone-division technology, the principle of FEI, tripping strategy, wide area communication and so on. Then from the view of engineering application, several issues need to be solved when researching the principle of FEI is proposed.

The paper introduces several new judging principles of FEI. These methods have excellent performance in reducing the dependence on wide area synchronous sampling, improving the sensitivity of backup protection.

This paper presents the limited wide area backup protection based on the inter-substation communication channel such as the pilot channel. This protection scheme can identify the fault element correctly simplify the setting and the coordination of the backup protection, and improve the tripping speed. In addition, the limited wide area backup protection can speed up the tripping of remote protection under the condition that the main power supply of the next substation protected is lost and achieve the fast fault isolation remotely.

\section{Funding \\ This work is supported by National Natural Science Foundation of China Science Foundation of China (No. 50377031 and No.50837002).}

\section{Authors' contributions}

$X G Y$ contributed to the conception of the study. ZZ contributed significantly to analysis and manuscript preparation; ZXL helped perform the analysis with constructive discussions. XWQ performed the analyses and wrote the manuscript; WBC conceived of the study, and participated in its design and coordination and helped to draft the manuscript. QG revised the manuscript. All authors read and approved the final manuscript.

\section{Competing interests}

The authors declare that they have no competing interests.

Received: 15 June 2016 Accepted: 1 August 2016

Published online: 11 August 2016

\section{References}

1. Novosel, D., Bartok, G., Henneberg, G., et al. (2010). IEEE PSRC report on performance of relaying during wide-area stressed conditions [J]. IEEE Transactions on Power Delivery, 25(1), 3-16.

2. Chen, D. (2004). Preliminary research on security protection technology of large-scale power grid [J]. Power System Technology, 28(9), 14-17. 27(in (hinese).

3. Working group c-6, system protection subcommittee. (2002). Wide Area Protection and Emergency Control [R]. IEEE PES power system relaying committee.

4. Xiang-gen, Y. I. N., Yang, W. A. N. G., \& Zhe, Z. H. A. N. G. (2010). Zonedivision and tripping strategy for limited wide area protection adapting to smart grid [J] (Proceedings of the CSEE, pp. 1-7). in Chinese.

5. Xin-zhou, D. O. N. G., \& Lei, D. I. N. G. (2009). Research on design of digital integrated protection and control system [J]. Power System Protection and Control, 01, 1-5. in Chinese.

6. Zhen-xing, G., Chuang-xin, G., Bin, Y. U., et al. (2011). Study of a fault diagnosis approach for power grid with information fusion based on multi-data resources [J]. Power System Protection And Control, 39(6), 17-23. in Chinese.

7. The editorial board. (2014). China Electric Power Encyclopedia [M]. Beijing: China Electric Power Press.

8. Cholley P, Crossley P, Van Acker V, et al. (2001). System Protection Schemes in Power Networks [J]. Cigre Technical Brochure.

9. Ji-xiu, Y. (2007). Wide area protection and emergency control to prevent large scale blackout [M]. beijing: China electric power press.

10. Cai, Y., Wang, L., Morison, K., et al. (2004). Current status and prospect of wide-area protection (dynamic stability control) technologies [J]. Power System Technology, 28(8), 20-25. in Chinese.

11. Jun, Y. I., \& Xiao-xin, Z. (2006). A survey on power system wide-area protection and control [J]. Power System Technology, 30(8), 7-12. 30(in Chinese).

12. Standardization administration of China. (2011). GB/T-26399-2 Technical guide for electric power system security and stability control [S]. SAC.

13. Jixiu, Y. (1999). Planning and application of power system security and stability control [J]. Electric Power, 32(5), 29-32. in Chinese.

14. Rockefeller, G. D., Wagner, C. L., Linders, J. R., et al. (1988). Adaptive transmission relaying concepts for improved performance [J]. Power Delivery IEEE Transactions on, 3(4), 1446-1458.

15. Xian-zhong, D., Zeng-li, Y., \& Xiao, C. (2005). Performance analysis of relay settings determined according to Off-line calculation and on-line calculation [J]. Automation Of Electric Power Systems, 29(19), 58-61. in Chinese.

16. Cao, G., Cai, G., \& Wang, H. (2003). Problems and solutions in relay setting and coordination [J]. Proceedings Of The Chinese Society For Electrical Engineering, 23(10), 51-56. in Chinese.

17. Orduna, E., Garces, F., \& Handschin, E. (2003). Algorithmic-knowledge based adaptive coordination in transmission protection [J]. IEEE Power Engineering Review, 22(12), 63. 
18. Ying, L. U., \& Boming, Z. (2007). Online relay setting check based on computer cluster []]. Automation Of Electric Power Systems, 31(14), 12-16. 106(in Chinese).

19. Songtao, Q., Chao, H., Falin, Z., et al. (2013). Development of the on-line verification system for Guangxi power grid relay settings []]. Southern Power System Technology, 4, 83-87. in Chinese.

20. Tejun, Z., Jian, Q., Chunyi, W., et al. (2015). Application of visualization technology based on SVG in on-line relay settings verification system [J]. Power System Protection and Control, 16, 112-117. in Chinese.

21. Jun, X., Dongyuan, S., Zengli, Y., et al. (2007). An on-line verification of relay settings and early warning system of protective relaying based on MAS [J]. Automation Of Electric Power Systems, 37(13), 77-82. in Chinese.

22. Youhuai, W., Zengli, Y., Hubing, Z., et al. (2015). Development and application of online verification and early-warning system for protective relay [J]. Proceedings of the CSU-EPSA, 27(6), 91-97. in Chinese.

23. Serizawa, Y., Myoujin, M., Kitamura, K., et al. (1998). Wide-area current differential backup protection employing broadband communications and time transfer systems [J]. IEEE Transactions on Power Delivery, 13(4), 1046-1052.

24. Hui-ming, X. U., Tian-shu, B. I., Shao-feng, H., et al. (2007). Study on wide area measurement system based control strategy to prevent cascading trips [J]. Proceedings Of The Chinese Society For Electrical Engineering, 27(19), 32-38. in Chinese.

25. Lim, S. I., Liu, C. C., Lee, S. J., et al. (2008). Blocking of zone 3 relays to prevent cascaded events [J]. IEEE Transactions on Power Systems, 23(2), 747-754.

26. Tian-qi, X. U., Xiang-gen, Y., Da-hai, Y., et al. (2009). Analysis on functionality and feasible structure of wide area protection system []]. Power System Protection And Control, 37(3), 93-97. in Chinese.

27. Li, Z., Yin, X., Zhang, Z., et al. (2011). Study on system architecture and fault identification of zone-division wide area protection [J]. Proceedings of the CSEE, 31(28), 95-103. in Chinese.

28. Zhenxing, L. I., Xianggen, Y., Zhe, Z., et al. (2010). Zone division and implementation on limited wide area protection system [J]. Automation of Electric Power Systems, 34(19), 48-52. in Chinese.

29. Wei, C., Zhen-cun, P., Jian-guo, Z., et al. (2006). A wide area protective relaying system based on current differential protection principle [J]. Power System Technology, 30(5), 91-95. 110(in Chinese).

30. Zhao-hui, C., Man-yong, Z., Hong-yang, Z., et al. (2009). Research and implementation of network protection system based on integrated and wide area information [J]. Power System Protection And Control, 37(24), 106-108. 113(in Chinese).

31. Yang, Z., Shi, D., \& Duan, X. (2008). Wide-area protection system based on direction comparison principle [J]. Proceedings Of The Chinese Society For Electrical Engineering, 28(22), 87-93. in Chinese.

32. Lin, X., Li, Z., Wu, K., et al. (2009). Principles and implementations of hierarchical region defensive systems of power grid [J]. Power Delivery IEEE Transactions on, 24(1), 30-37.

33. Yang, W., Xianggen, Y., Zhe, Z., et al. (2010). Wide area protection based on genetic information fusion technology [J]. Transactions Of China Electrotechnical Society, 25(8), 174-179. in Chinese.

34. Zhen-xing, L. I., Xiang-gen, Y. I. N., \& Zhe, Z. H. A. N. G. (2011). A new algorithm of wide area protection on multi-information fusion [J]. Automation of Electric Power Systems, 09, 14-18. in Chinese.

35. He, Z., Zhang, Z., Chen, W., et al. (2011). Wide-area backup protection algorithm based on fault component voltage distribution [J]. IEEE Transactions on Power Delivery, 26(4), 2752-2760.

36. Zhi-hui, D., \& Zeng-ping, W. (2010). Overview of research on protection reliability [J]. Power System Protection And Control, 38(15), 161-167 (in Chinese).

37. Hua, W. A. N. G., Zhe, Z. H. A. N. G., \& Xiang-gen, Y. I. N. (2011). A wide area protection algorithm based on fault voltage distribution [J]. Automation of Electric Power Systems, 07, 48-52. in Chinese.

38. Jiale, S., Kai, L., Xiaohua, S. U., et al. (2008). Novel transmission line pilot protection based on integrated impedance [J]. Automation Of Electric Power Systems, 32(3), 36-41. in Chinese.

39. Zhen-xing, L. I., Xiang-gen, Y. I. N., \& Zhe, Z. H. A. N. G. (2010). A study of wide-area protection algorithm based on integrated impedance comparison [J]. Transactions of China Electrotechnical Society, 08, 1-5. in Chinese.

\section{Submit your manuscript to a SpringerOpen ${ }^{\circ}$ journal and benefit from:}

- Convenient online submission

- Rigorous peer review

- Immediate publication on acceptance

- Open access: articles freely available online

- High visibility within the field

- Retaining the copyright to your article

Submit your next manuscript at $\gg$ springeropen.com 